\title{
The filamentary structure of ELMs in the scrape-off layer in ASDEX Upgrade
}

A. Herrmann, A. Kirk ${ }^{1}$, A. Schmid, B. Koch², M. Laux, M. Maraschek, H.W. Mueller, J. Neuhauser, V. Rohde, E. Wolfrum, ASDEX Upgrade team

Max-Planck-Institut für Plasmaphysik, Boltzmannstr. 2, D-85748 Garching,Greifswald, Germany

${ }^{I}$ EURATOM/UKAEA Fusion Association, Culham Science Centre, Abingdon, UK

${ }^{2}$ Humboldt - Universität zu Berlin. Institut für Physik, Experimentelle Plasmaphysik, Germany

\begin{abstract}
The filamentary structure in the SOL of ASDEX Upgrade during type-I ELMs was investigated by combined measurements of reciprocating Mach probe, filament probe - a combination of Langmuir probes and magnetic sensor for the radial component of the magnetic field, and IR-thermography of the reciprocating probe head. A correlation along field lines between the Langmuir probes was found, confirming the field aligned structure of the observed filaments. The ELM radial e-folding lengths of particle and heat flux are comparable (about $2 \mathrm{~cm}$ ). This may be explained by an approximately constant ion temperature well above the electron temperature in the far SOL. The ion temperature estimated from the comparison of heat and particle flux is about $30-60 \mathrm{eV}$.
\end{abstract}

PSI $17^{\text {th }}$ keywords: ELMs, Fluctuation and turbulence, ASDEX Upgrade

JNM keywords: PO500 Plasma-materials interaction,

LO200 Limiter materials,

FO400 First-wall materials

PACS: $52.40 . \mathrm{H}, 52.55 \mathrm{Fa}$

mail: albrecht.herrmann@ipp.mpg.de

phone: +498932991388

fax: $\quad+498932992580$

address: Max-Planck-Institut für Plasmaphysik

Boltzmannstr. 2

D-85748 Garching 


\section{Introduction}

The envisaged plasma parameters for the operation of the next step experiment on the way to a burning fusion reactor, ITER, are based on a transport barrier at the plasma edge resulting in a high confinement (H-mode) regime. Up to now this H-mode regime has been associated with MHD-type relaxation instabilities in the plasma edge (Edge localized modes - ELMs) that release a measurable portion of the confined plasma energy.

The fraction of plasma energy lost per type-I ELM event increases with energy confinement, i.e. with the size of the experiment. In ASDEX Upgrade up to $3 \%$ of the total plasma energy is lost per ELM. The losses in the largest running fusion experiment, JET, are in the order of $10 \%$ [1] of the total plasma energy. As for the inter-ELM period, most of the ELM energy, lost preferentially in the outer mid-plane, is deposited in the divertor along the strike lines, and only a fraction of the mid-plane loss is transported to the main chamber walls [1]. Nevertheless, the first wall is not designed to receive the potentially high local heat loads during ELMs. The understanding and/or parameterisation of the first wall load is essential for the design of a next step reactor.

Present ELM models and simulations, e.g. MHD and/or turbulence codes, show that the release of the ELM energy is not axisymmetric but localized in the outer mid-plane in the form of weakly correlated helical events. Their toroidal distribution is usually characterized by a toroidal quasi-mode number. The corresponding localized helical loss of particles and energy from the hot pedestal appears in the scrape-off layer in the form of magnetic field aligned filament-like plasma structures. These structures have been detected and investigated by various diagnostics in different experiments; see e.g. main chamber filaments in the $\mathrm{H}_{\alpha}$ light at MAST $[2,3]$ and the spiral power deposition structures on the upper divertor targets of ASDEX Upgrade [4]. With this background, the burst like structure of signals measured in the outer plasma edge during ELMs by Langmuir probes or retarding field analyzer [5] is interpreted as the foot print of moving filaments [6]. Whereas the existence of the filamentary field aligned structure in the SOL during ELMs seems to be proved by experiments, the crucial question for plasma wall interaction is the energy content of such filaments and the loss mechanisms, which has only started to be answered [5,7]. In addition to numerical simulations, analytical and simple heuristic models have been proposed which need experimental input for validation. 
This paper presents results from simultaneous measurements with reciprocating Langmuir probes, partly with incorporated magnetic pick-up coils and thermography of the reciprocating probe head. The results contribute to the following questions:

- Is the energy content of the filaments reduced while travelling through the SOL or is it constant because the filament is attached to the central plasma column?

- Is there any relation between the measured ion saturation current and heat load deposition?

- Are the Langmuir signals correlated with magnetic perturbations as measured by the magnetic pick-up coils?

\section{Experiments}

The ASDEX Upgrade tokamak [8] is well suited for the investigation of plasma wall interaction due to its flexible heating methods and diagnostics capability. The results presented in this paper are from combined measurements with different Langmuir and magnetic pick-up probes in the far scrape-off layer (SOL) and with thermography of the inner wall.

An overview on the arrangement of the Langmuir and magnetic pick-up probe as well as the field of view of the ir-camera is shown in Fig. 1. A Mach type reciprocating probe was used to measure the ion saturation current in the far SOL outside any limiter shadow with $2 \mathrm{MHz}$ sampling rate. Simultaneously, the temperature of the probe head was recorded by a high speed infra-red (ir)-camera [9]. The settings of the camera were optimized to get sufficient spatial resolution $(2 \mathrm{~mm})$ and a good time resolution of $100 \mu \mathrm{s} /$ frame in order to allow the detection of the evolution of filaments during an ELM. This is a significant improvement compared to earlier measurements [7] where the filament detection was mainly stroboscopic because the frame rate of the camera was comparable to the ELM duration. Subsequently, the heat flux was calculated from the measured surface temperature evolution at the probe head by the THEODOR code [10].

A 'filament probe' (see Fig. 1) was installed to measure the rotation velocity of filaments by measuring the time delay between the separated Langmuir pins and simultaneously to measure the radial component of the magnetic field. The probe can be moved $3.5 \mathrm{~cm}$ radial via a magnetic force (magnetic coil in the toroidal field, time of movement about $200 \mathrm{~ms}$ ) to be positioned radially inward of the protection limiter. It is placed $0.6 \mathrm{~m}$ toroidally away from the reciprocating probe head, and $1 \mathrm{~cm}$ radially behind the front edge of the 
reciprocating probe. The poloidal position of the filament probe is selected such that a field line starting in the centre of the reciprocating probe is connected to the filament probe for values of the safety factor between $\mathrm{q}_{95}=3.2$ and 7 (pitch angle variation of $\pm 3.5^{\circ}$, see Fig. $1)$.

Type-I ELMy H-mode discharge scenarios were used for the measurements. A plasma shape with a relatively small cross section allows the radial distance of the outboard separatrix to the active ICRH limiter at $\mathrm{R}=2.2 \mathrm{~m}$ to be increased from $3.5 \mathrm{~cm}$ to $11 \mathrm{~cm}$ without touching the inner column. During the radial movement of the plasma, the radial positions of the movable probes (reciprocating and filament probe) were kept fixed. The reciprocating probe was positioned in front of the active ICRH limiter. The filament probe was outside the protection limiter in direct magnetic connection to the reciprocating probe. The timing of probe and plasma movement was kept constant for all discharges. Global parameters were varied as follows: the toroidal magnetic field was set to $B_{t}=-2$ and $-3 T$, the plasma current to $I_{p}=0.8$ and $1 \mathrm{MA}$, respectively. This results in a variation of the safety factor $\mathrm{q}_{95}$ between 3.5 and 6.5. The heating power was increased from $5 \mathrm{MW}$ for $\mathrm{B}_{\mathrm{t}}=-2 \mathrm{~T}$ to $6.6 \mathrm{MW}$ for $\mathrm{B}_{\mathrm{t}}=-$ $3 \mathrm{~T}$ to keep the plasma stored energy constant at about $500 \mathrm{~kJ}$. The line averaged density was $60 \%$ of the Greenwald density.

\section{Results and Discussion}

The signals gained from the combined measurement during a single ELM are shown in Fig. 2. The Langmuir probe measurements show a burst like structure of the ion saturation current with a width of the bursts of a few tens of microseconds and a period of a few hundred microseconds (Fig. 2). Each burst is attributed to a single filament. The temporal evolution of a burst is characterized by a steep increase and a slower decrease on a time scale of tens of microseconds with the tendency to become broader over the ELM lifetime as indicated by the grey stripes in Fig. 2. These single filaments still show a substructure of short spikes with each up to 10 microseconds width. Signatures corresponding to a single filament are found on each signal. Three of them are marked by grey bars in Fig. 2. Taking into account the time resolution of $100 \mu \mathrm{s}$, it is obvious that the heat flux to the probe head follows the ion saturation current measured with the reciprocating probe. Here the mean value for the heat flux near to the leading edge of the probe head is plotted. The lateral variation of the heat deposition to the probe head is expressed by the standard deviation of 
the signal (dashed lines in Fig. 2) which is highest during the early phase of an ELM with the strongest filaments.

Both, the Langmuir pins at the reciprocating probe and at the filament probe which are connected by magnetic field lines show a good correlation of the base temporal structure (filaments) as expected for field aligned structures. However, the fine structure (shape and strengths of the spikes) differs between both signals. In addition, there is a delay of $20 \mu \mathrm{s}$ between the onset of the first filament at the filament probe with respect to the reciprocating probe. This time delay and the slightly different radial position $(1 \mathrm{~cm})$ can be used to estimate the order of magnitude for the radial velocity of this filament of $v_{r} \approx 500 \mathrm{~m} / \mathrm{s}$. It should be mentioned here, that the clear identification of individual filaments is not always obvious, in particular during the late phases of an ELM and needs more sophisticated methods. The Mach number is usually well below 1 and shows a change correlated with the filaments. Here, negative Mach numbers means a flow towards the lower outer divertor.

A correlation with the magnetic signal is obvious for a few filaments only. It shows the tendency to become weaker over the ELM lifetime. In particular, the magnetic signal strength differs from the amplitude of the measured $j_{\text {sat }}$ as can be seen by comparing the signals in the first two grey bars for the magnetic and the filament probe.

The heat flux to the probe and the corresponding particle flux can be used to estimate the ion temperature. For convection dominated heat transfer to the target, the heat load is given by the impinging particle flux (ion saturation current) and the energy deposited per particle $\left(\mathrm{T}_{\mathrm{i}}\right.$, $\mathrm{T}_{\mathrm{e}}$ ). The effect of the sheath in front of the target on the acceleration of ions and deceleration of electrons as well as the secondary electron emission, $\gamma_{\mathrm{e}}$, is considered in the sheath transmission factor, $\gamma$ (see e.g. equ. 9.2.12 in [11].

$q_{\|}=\gamma \frac{j_{\text {sat }}}{e} T_{e}$ with $\gamma=\frac{2}{1-\gamma_{e}}+\frac{2 T_{i}}{T e}-0.5 \ln \left(\frac{2 \pi m_{e}}{m_{i}}\left(1+\frac{T_{i}}{T_{e}}\right) \frac{1}{\left(1-\gamma_{e}\right)^{2}}\right)$.

Eq.: 1

Using the data from Fig. 2 the product of sheath transmission factor and electron energy become: $q_{\|} e / j_{s a t}=\gamma T_{e} \approx 100 \div 200 \mathrm{eV}$ (Fig. 2).

The measurements are done in the far SOL where the electron temperature as measured by laser scattering [12] is much smaller compared to the ion temperature. Under these conditions, the ions dominate the heat deposition and the sheath transmission factor is about $\gamma=3 \times \frac{T_{i}}{T_{e}}$ resulting in an ion temperature of about $30-60 \mathrm{eV}$ and a density in the filament of 
$4 \div 6 \times 10^{19} \mathrm{~m}^{-3}$ at a separatrix distance of $4 \mathrm{~cm}$ which is about the pedestal density in this shot. Two main effects cause a systematic error on this estimation of the ion temperature. The heat flux is measured $5 \mathrm{~mm}$ radially behind the position of the Langmuir pin (see Fig. 1) which results in an underestimation of the ion temperature. Surface effects influencing the surface temperature measurement and the heat flux calculation tends to overestimate the calculated heat flux. Due to this systematic error no attempt was made to derive the ratio of ion to electron temperature from an accurate measurement of the sheath transmission factor. A robust figure which is independent on systematic errors in the measurement of the absolute values is an e-folding length. The radial ELM e-folding lengths of particle and heat flux were derived from the signal decay during the radial sweep of the plasma and were used to get information on the mechanism of heat transport and the temperature ratio in the far SOL of ASDEX Upgrade as discussed in the next paragraph.

For 6 discharges the decay of the heat load and $\mathrm{j}_{\mathrm{sat}}$ was measured as shown for one discharge in Fig. 3. In this figure the maximum value of each ELM event was plotted. As can be seen in this figure, the e-folding lengths of the ion saturation currents measured with the two different Langmuir probes (filament and reciprocating probe, see Fig. 1) and that of the heat flux are comparable. This is a typical feature for all the discharges under investigation as is illustrated in Fig. 3 (inset). This is not trivial because typically also the temperature of the particles might change with the radial distance to the separatrix. The fact that $\lambda_{\mathrm{q}}$ is equal to $\lambda_{\text {jsat }}$ indicates $\lambda_{T i}>\lambda_{n}$, i.e. both, the e-folding length for particle and heat flux is dominated by the density e-folding length. From this follows that the reduction of the energy content in the filament is rather a loss of particles than a cooling of ions or electrons. This requires that the heat conduction $\left(\sim T_{e}^{7 / 2}\right)$ has to be small compared to convective losses $\left(\sim n_{e} T_{i}^{3 / 2}\right)$ as expected for cold electrons $(<10 \mathrm{eV})$ and marginally collisional ions in the far SOL (Separatrix distance $>40 \mathrm{~mm}$ ) of ASDEX Upgrade.

As can be seen from Fig. 2, the dynamic of the filaments during a single ELM is high and the amplitude ratio between the filaments is different for each Langmuir pin. These varying amplitudes of the filaments are visualized in Fig. 4. Here, the amplitude of the first 4 filaments of each ELM is plotted vs the distance to the separatrix. The resulting e-folding length is comparable to that derived for the decay of the maximum alone (Fig. 3). This means that on average the filaments, regardless of size, are damped with the same rate, although with significant variability on an individual basis. This holds over the whole radial 
range as expressed by the enveloping lines in Fig. 4. For a given radial distance, the data points represent the temporal distribution of the filament strength during a single ELM. It is obvious that the largest filament is not necessarily the first filament in a single ELM. It can also occur later in an ELM. Whether or not the distribution of the largest filament is arbitrary or is more dominant for certain ELM phases has to be investigated by statistical methods.

\section{Summary}

The filamentary structure in the SOL of ASDEX Upgrade during type-I ELMs was investigated by combined measurements of different Langmuir probes, magnetic sensors for the radial component of the magnetic field, and thermography of the Langmuir probe head. A correlation along field lines between the Langmuir probes, $0.6 \mathrm{~m}$ separated from each other, was found. This confirms the field aligned structure of the observed filaments. But, still the fine structure of a single filament is different. The correlation between the fast Langmuir probe measurements and the high temporal resolution ir-measurements reveals that the measured ion saturation current is related to heat deposition and that in particular the short events of a few tens of microseconds deposit a significant amount of heat to the probe head.

The heat flux calculated from the ion saturation current with the assumption of an ion dominated sheath and an ion temperature of $30-60 \mathrm{eV}$ is comparable to the ir-measurement. Radial scans results in an e-folding length of about $2 \mathrm{~cm}$ for both the heat and the particle flux. The fact that the particle and the heat flux have the same e-folding length can be explained by a constant ion and electron temperature and mostly convective parallel transport. This finding is consistent with an ion temperature of $30-60 \mathrm{eV}$. The far SOL ion energy as derived from the combined measurement is about a factor of ten below the pedestal ion energy of about $800 \mathrm{eV}$.

The dynamic range of the filament strength during an ELM is about 10 ( $\mathrm{max} / \mathrm{min})$ and is sustained during the radial scan. There is no preferential damping of strong filaments.

\section{References}

[1] A. Herrmann, T. Eich, S. Jachmich, et al., J. Nucl. Mater. 313-316 (2003) 759.

[2] A. Kirk, H. R. Wilson, R. Akers, et al., Plasma Phys. Control. Fusion 47 (2005) 315-333.

[3] B. Koch, This conference (2006).

[4] T. Eich, A. Herrmann, J. Neuhauser, et al., Plasma Phys. Control. Fusion 47 (2005) 815-842.

[5] R. A. Pitts, W. Fundamenski, K. Erents, et al., Nucl. Fusion 46 (2006) 82-89. 
[6] A. Kirk, T. Eich, A. Herrmann, et al., Plasma Phys. Control. Fusion 47 (2005) 995-1013.

[7] A. Herrmann, T. Eich, V. Rohde, et al., Plasma Phys. Control. Fusion 46 (2004) 971-979.

[8] A. Herrmann, O. Gruber, Fusion Science and Technology 44 (2003) 569-577.

[9] High speed CMT camera; Vol. www.thermosensorik.de.

[10] A. Herrmann, ASDEX Upgrade Team, Europhysics Conference Abstracts (CD-ROM, Proc. of the 28th EPS Conference on Controlled Fusion and Plasma Physics, Madeira 2001) 25A (2001) 2109-2112.

[11] J. Wesson Tokamaks, 3 ed.; Clarendon Press-Oxford: Oxford, 2004.

[12] J. Neuhauser, D. Coster, H. U. Fahrbach, et al., Plasma Phys. Control. Fusion 44 (2002) $855-$ 870.

\section{Figures}

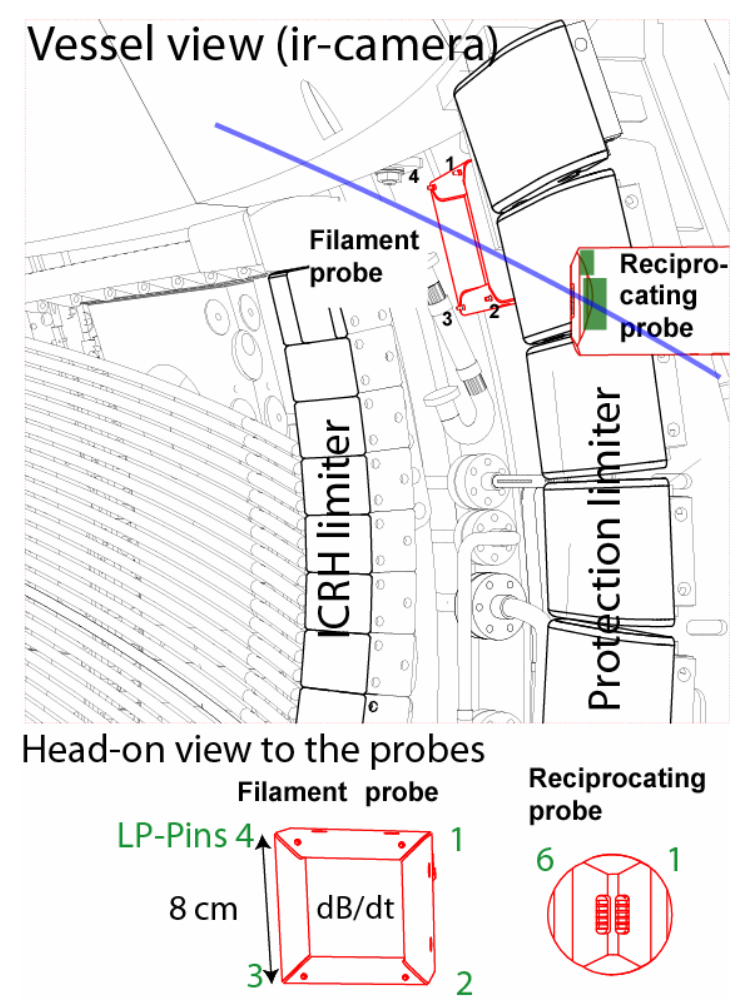

Fig. 1 Langmuir and magnetic pick-up probes in the field of view of the ir-camera located above the outer mid-plane of ASDEX Upgrade. The regions which were used for the heat flux plots and the names of the probes are marked. The line indicates a magnetic field line. The toroidal separation of the probes is about $0.6 \mathrm{~m}$. 


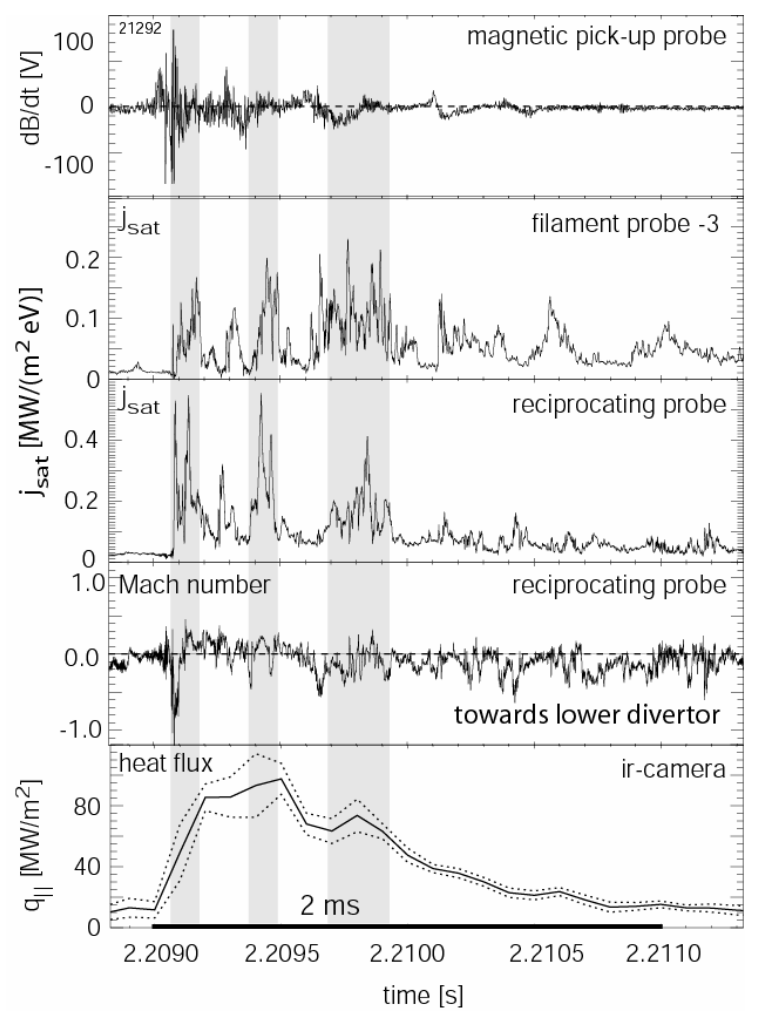

Fig. 2 Time slices of measurements during a single ELM with the probes from Fig. 1. The Mach number was calculated from pin 1 and 6 of the reciprocating probe. The heat flux is the mean value of the center region at the probe head with the dashed lines showing the standard deviation. The grey bars mark single filaments for better comparison.

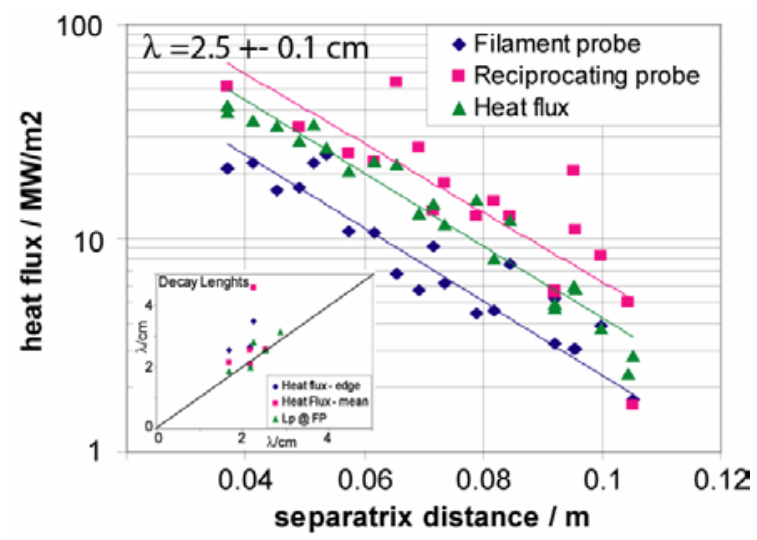

Fig. 3 Heat flux decay lengths as measured by Langmuir probes at two different toroidal positions and by thermography. A factor of $\gamma T_{e}=100 \mathrm{eV}$ is assumed to derive the heat flux from the ion saturation current. The inset shows all e-folding lengths measured by the filament probe and by thermography vs. the reciprocating probe results. 


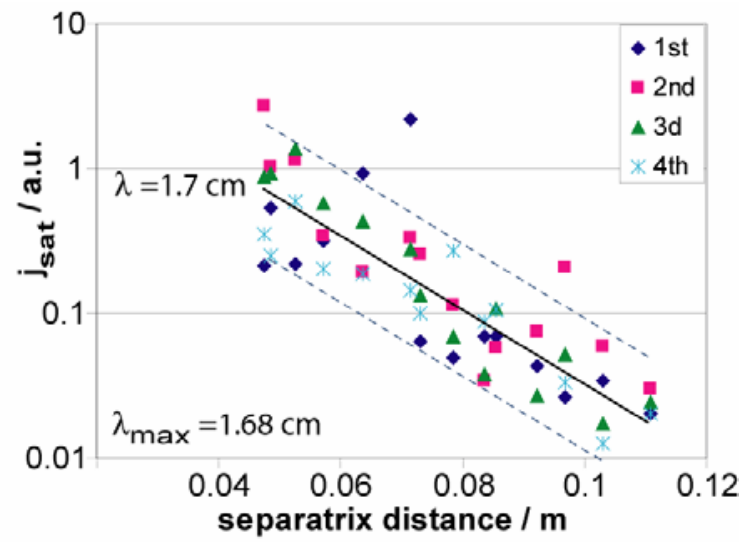

Fig. 4 E-folding lengths for the first 4 filaments in an ELM as measured with the reciprocating probe. 